\title{
A Study on Improvement of Route Plan by Using the Approximate Model Made by pSeven Big Data Technology
}

\author{
Chao.Chen, Jeong-Jin.Lee, Dae-Han.Choi, and Sang-Su.Byeon
}

\begin{abstract}
In this paper, pSeven big data technology will be used to analyze the data of ship operation, approximate model of key factors related to ship navigation energy efficiency can be derived. For improving route plan considering energy efficiency, the approximate model can be used to evaluated route plans and then determine which one is the optimum choice. By using ECO panel function of Transas Ship Handling Simulator, these models were verified.
\end{abstract}

Keywords-Approximate Model, Big Data Technology, Eco function of Simulation, Improvement of Route Plan, Navigation Efficiency.

\section{INTRODUCTION}

$\mathrm{T}$ HE global economy and the international shipping industry showed "Two Low" properties, that is to say, global economic growth is low, and meanwhile the international shipping market is also depressed[1]. Shipping companies are facing more and more serious challenges. Predicament forced change, and crisis breeds innovation[2]. So shipping companies needs to cultivate new competitive advantages and build new survival and development capabilities.

Big data technology is now widely used in production, marketing, science research and logistics and other fields, and is seen as another revolution since Internet. The strategic significance of large data lies in the specialization of the correlation of massive data, accurate analysis of users' preferences, scientific adjustment of their products, and tapping new business opportunities and creating new values. The future development of shipping companies will be more and more dependent on information flow, and the core of information flow is big data. Shipping companies have great prospects of research and development of big data. Big data will be a very important factor in determining the core competitiveness of enterprises in the future.

pSeven is bigdata analysis tool which is a powerful design space exploration platform for automation of engineering

Chao.Chen is Senior Research Engineer of Marine Electronics Co. 48792 South Korea, (e-mail: chenchao@mecys.com).

Jeong-Jin Lee is Senior Research Engineer of Marine Electronics Co. 48792 South Korea, (e-mail: jjlee@mecys.com). simulation and analysis, multidisciplinary optimization and data mining.[3]

In this paper, pSeven will be used to analyze the navigational data gathered from a single merchant ship(container ship) belong to a big Korean shipping company. Sensitivity among gathering data were analyzed, and influence power of the factors were decided, and then key factors related to energy efficiency of vessel navigation can be analyzed. Analysis method is to use approximate model using math models to predict values comparing with actual data. By using the approximate models related to rate of fuel consumption, route plan of merchant vessels can be improved.

In section 2, analysis of navigation big data was introduced. In section 3, approximate model was generated and used to predict. In section 4, conclusions are summarized.

\section{ANALYSIS OF NAVIGATION BIG DATA}

\section{A. Gathering and Data Organization}

Navigation data usually were gathered not only from data records of all kind of sensors installed in a vessel and handwriting/electronical format navigation log etc internal data, but also meteorological data, traffic notification etc external data. Usually all kinds of these data have different data format, a process of data organization for acquiring a universal data format was needed. In order to use statistical techniques, some non-digital format data need to be digitized and converted by dividing or replacing and a part of these data should be deleted if they are not difficult to be digitized. As a result, a universal table including 912 parameters was abstained as Fig.1 shows.

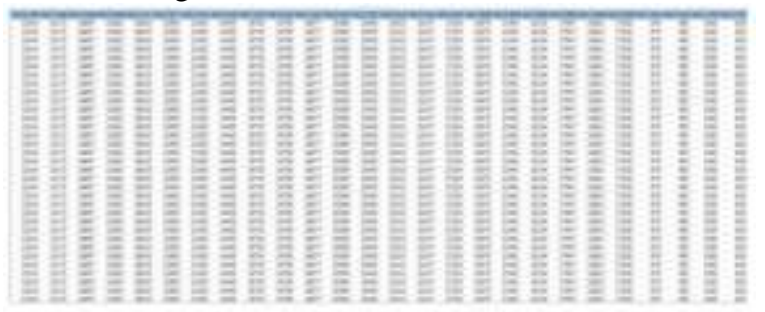

Fig. 1 Organized Universal Data Set

\section{B. Key Parameters Extraction}

Total 9 parameters (shown in TABLE I) were selected for evaluating the sensitivity of the parameter, the parameters 
which are most related to energy consumption will be selected according to evaluation values[4].

TABLE I

SELECTED DEPENDENT VARIABLE TABLE

\begin{tabular}{ll}
\hline \hline No. & \multicolumn{1}{c}{ Dependent Variable } \\
\hline 1 & STW \\
2 & SOG \\
3 & Engine Power \\
4 & RPM \\
5 & Ship fuel oil consumption \\
6 & Fuel oil consumption \\
7 & Daily Bunker Consumption \\
8 & Engine efficiency \\
9 & Fuel consumption per nautical mile \\
\hline
\end{tabular}

Sensitivity and Dependency Analysis(SDA) was used to evaluate the sensitivity and dependency among 912 independent variable parameters and the 9 dependent variable parameters.

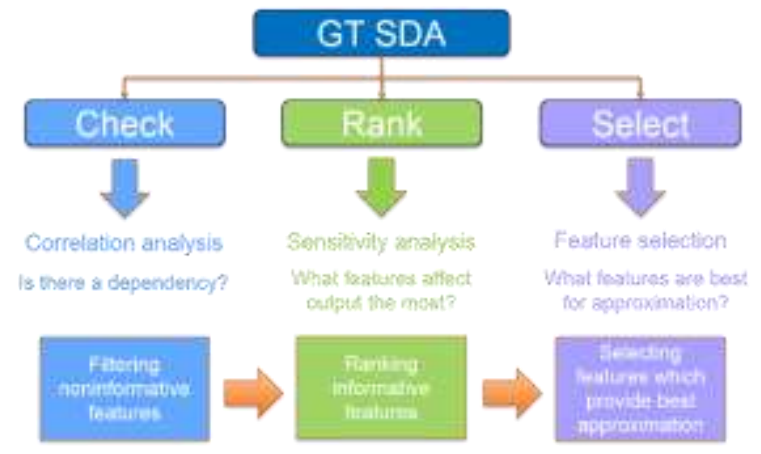

Fig. 2 Sensitivity and Dependency Analysis

In pSeven, the process of the evaluation is sample as Fig.3 shows.

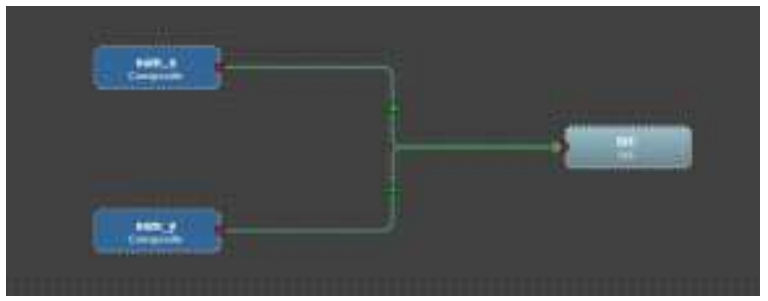

Fig. 3 Evaluation Process in pSeven

The results of the evaluation were shown as following figures. For research purpose, the voyage data were specially divided into sea voyage and harbor voyage

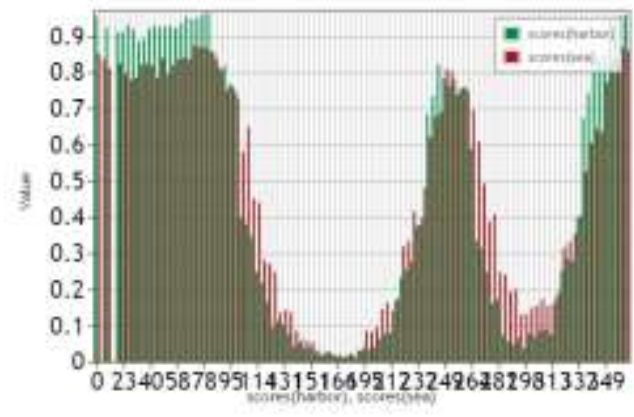

Fig. 4 STW SDA Result

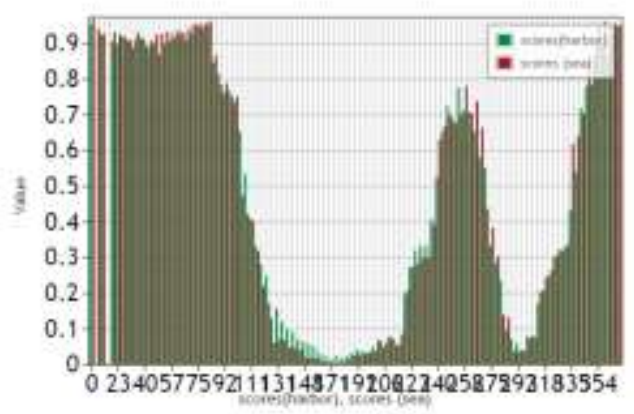

Fig. 5 SOG SDA Result

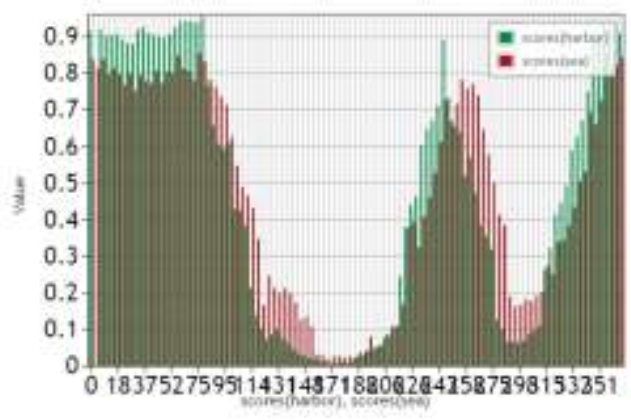

Fig. 6 RPM SDA Result

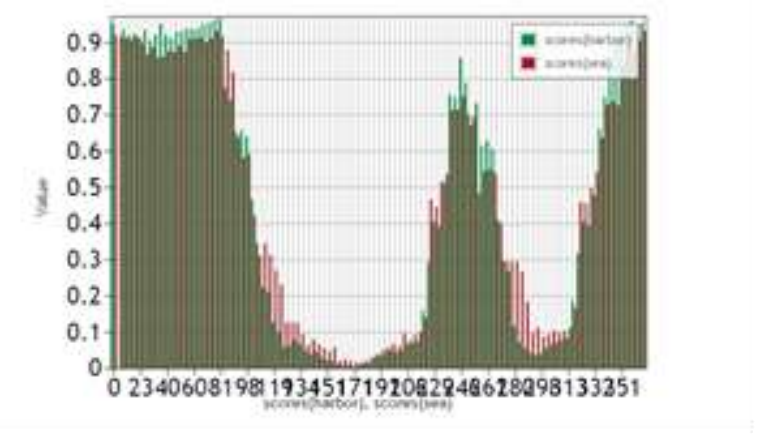

Fig. 7 Engine Power SDA Result

As the figures show, although the voyages were divided into harbor voyage and sea voyage, similar tendency of sensitivity and dependency results was shown. And parameters related actual navigation fuel consumption can be mainly divided as three clusters. IVE value 0.8 was used as the threshold for defining the key parameters. That is to say, when IVE values of an independent variable parameter bigger than 0.8 , it is a key parameter. The selected key parameters were shown in the Fig.8.

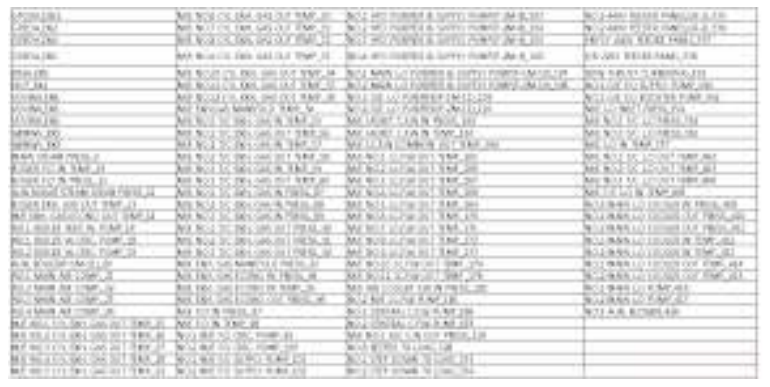

Fig. 8 Selected Key Parameters 


\section{APPROXIMATED MODEL}

\section{A. Approximate Model Generation}

When generating an approximate model, the most suitable model generation technique should be selected according to the complex factors such as the characteristics of each data and the number of variables.

Besides a wide range of well-known state-of-the-art approximation techniques (e.g., Splines, Linear Regression, Kriging, etc), original techniques like HDA(Higher Dimension Approximation, SGP-Sparse Gaussian Processes, Tensor Approximation and incomplete Tensor Approximation, Tensor Gaussian Processes etc. techniques were also provided in pSeven. It takes considerable time and cost to generate an approximate model by using one of these different techniques. So before generating approximate model, a proper approximation technique should be carefully selected. In the following figure, convergence comparison result of different techniques was shown.

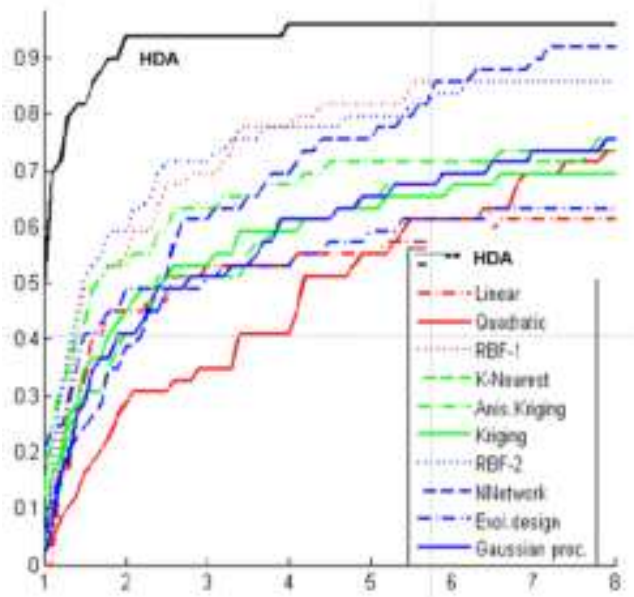

Fig. 9 Convergence Result of Main Approximation Techniques

Selecting a proper technique is different, however, a smart selection function is provided in pSeven. Flow chart of smart selection is shown in Fig.10.

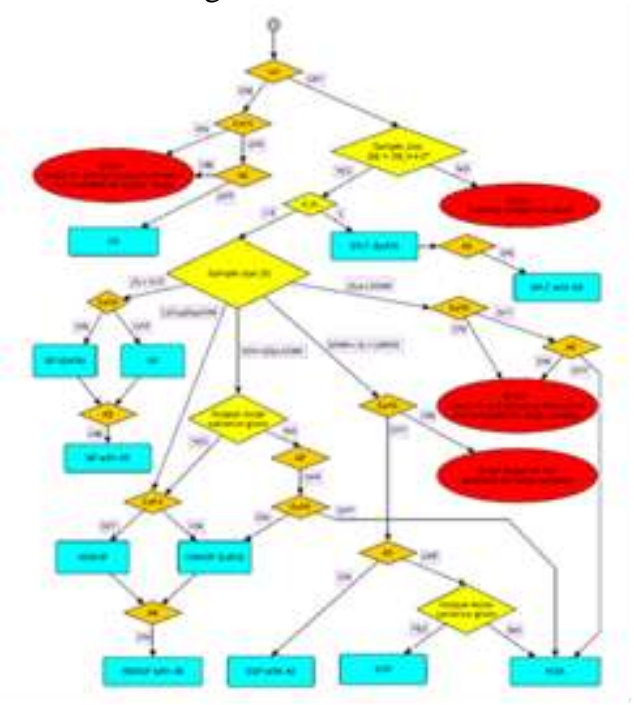

Fig. 10 Flow Chart of Smart Selection
In this case, after smart selection, HDA was selected to generate the approximate model. About 4,000 sampling data were used as training data during the generation process. After generation of model, in order to test the generated model, another 4,000 sampling data should be used to generate perdition data. Evaluation can be implemented between original data and perdition data. In Fig.11, results of training and testing were shown.

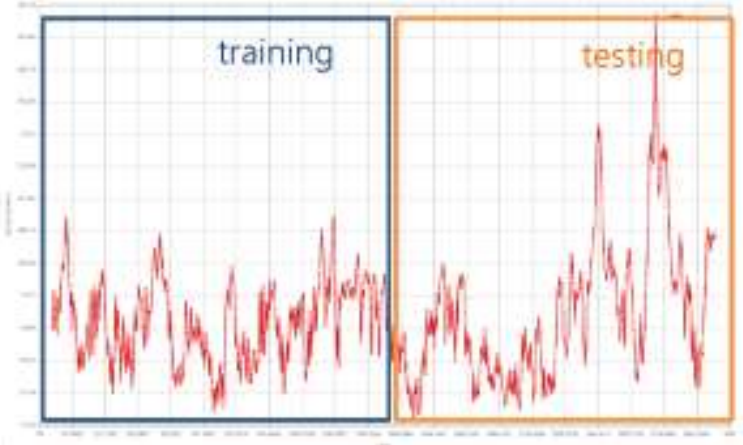

Fig. 11 Result of Training and Testing

\section{B. Comparison between Prediction Data and Original Data by} Using Approximate Model

For RPM in sea voyage and harbor voyage, the comparison results between prediction data and original data were shown in Fig. 12 and Fig.13

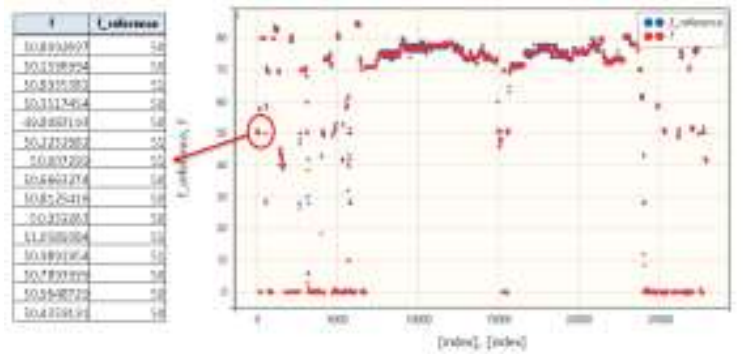

Fig. 12 RPM Comparison Result in Harbor Voyage

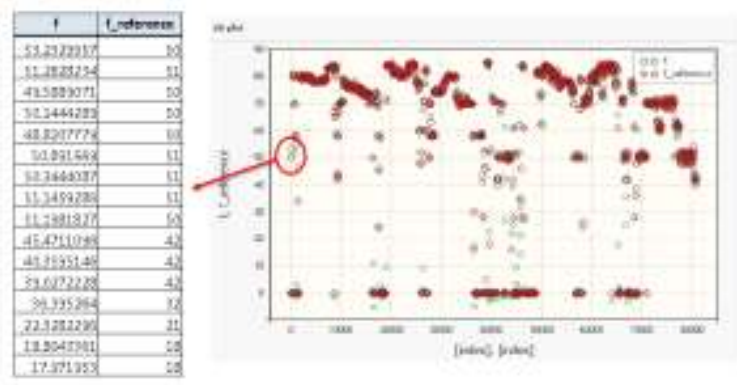

Fig. 13 RPM Comparison Result in Sea Voyage

As the results shows, the approximate model worked pretty well. And the approximate model can be more accurate if more training data were used into generation.

\section{Usage of Approximate Model}

$\mathrm{C} / \mathrm{C}++$ code of approximate model can be exported from pSeven. Then the code can be used into the navigational systems like ECDIS(Electronic Chart Display \& Information System), EEMS(Energy Efficiency Monitoring System), SMS(Ship Management System) etc. In this study, code of an approximate model related to fuel consumption was added into 
ECDIS. So when a second officer planed a route in ECDIS, prediction value of fuel consumption by using prediction function for the route can be supplied. Then the seaman can compare the fuel consumption of different route options. The most fuel saving route can be saved as an economical one. Also shipping companies not only can use this function to verify the summited routes are proper or not, but also can know in advance the roughly amount of fuel required for the planned voyage of the ship. As a result, the prediction function will help seaman improving route planning.

\section{Verification of Approximate Model}

Until now this prediction function is only in research phase, it is hard to test it on real ship, so it was verified in Transas ship handling simulator by using the ECO panel function of it. A similar container carrier model which importing approximate model was used for the testing. The result of the verification was quite positive.

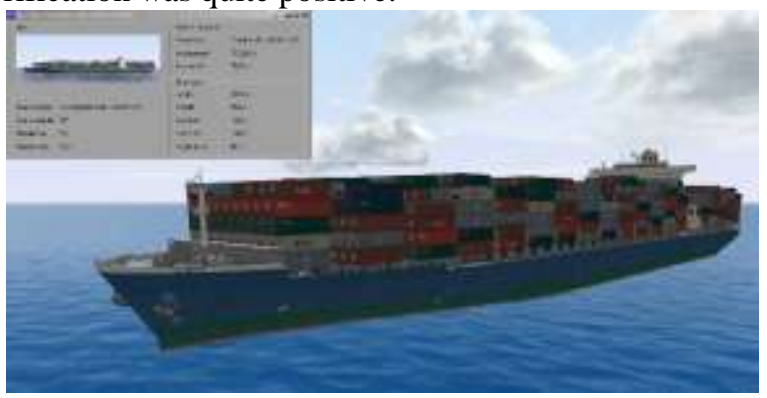

Fig. 14 Model Container Ship

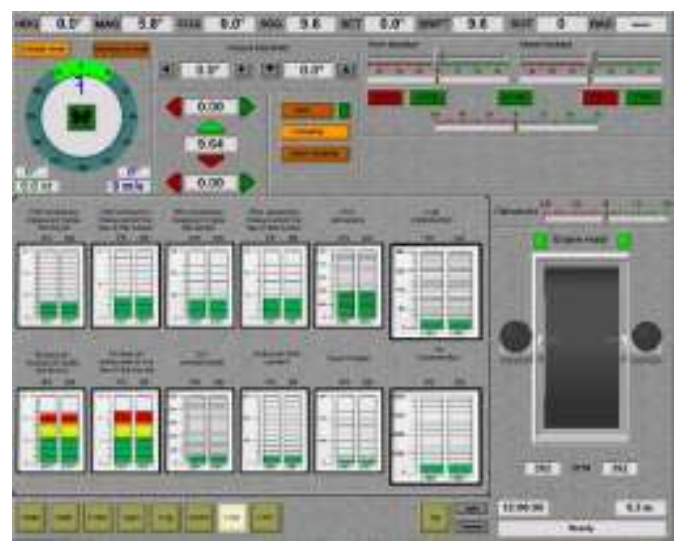

Fig. 15 ECO Panel

\section{CONCLUSION}

In this paper, in order to improve the fuel consumption of merchant ship, navigation big data were analyzed and an approximate model was generated by pSeven big data tool. Some conclusions are as follows.

1) Approximate model showed a tendency to follow the actual data.

2) The approximate model was applied into ECDIS as a prediction function of fuel consumption. Seaman can use the function for improving route planning. Results show the model is quite useful.

However, the model still need to be improved by continual research on the relationship between the parameters related to fuel consumption

\section{ACKNOWLEDGMENT}

This study is supported by the project (No.10051163) of Industry Core Development Projects of Ministry of Trade, Industry and Energy of South Korea Government. Thanks for the support to the study.

\section{REFERENCES}

[1] Jin-Seok.Oh, Development of a PCS Simulator and Data Storage System for Energy Saving in the Ship, Journal of the Korea Institute of Information and Communication Engineering, Vol. 19, No.9, 2015, 2189-2196.

[2] Yong-Dae.Kim, Jeong-Jin.Lee, Chao.Chen, Nam-Seon.Kang, A Research on Optimized and Safe Navigation Support System for Improving Ship Energy Efficiency Based on ECDIS, proceeding of Asia Navigation Conference 2016, 2016, pp.263-266.

[3] DATADVANCE, pSeven 6.10 User Manual

[4] Jeong-Hun.Sin, Jeong-Yeon.Sim, Jin-U.Park, Dae-Han.CHOI, SangSu.Byeon, A Study on the Improvement of Sailing Efficiency Using Big Data of Ship Operation, proceeding of 2017 Spring Conference of Korean Society of Marine Environment and Safety, 2017, pp.362. 